\title{
Traffic Classification using a Statistical Approach
}

\author{
Denis Zuev ${ }^{1}$ and Andrew W. Moore ${ }^{2}$ \\ 1 University of Oxford, Mathematical Institute, zuev@maths.ox.ac.uk* \\ 2 University of Cambridge, Computer Laboratory, andrew. moore@cl.cam.ac.uk ${ }^{\star \star}$
}

\begin{abstract}
Accurate traffic classification is the keystone of numerous network activities. Our work capitalises on hand-classified network data, used as input to a supervised Bayes estimator. We illustrate the high level of accuracy achieved with a supervised Naïve Bayes estimator; with the simplest estimator we are able to achieve better than $83 \%$ accuracy on both a per-byte and a per-packet basis.
\end{abstract}

\section{Introduction}

Traffi c classifi cation enables a variety of other applications and topics, including Quality of Service, security, monitoring, and intrusion-detection that are of use to researchers, accountants, network operators and end users. Capitalising on network traffi $\mathrm{c}$ that had been previously hand-classifi ed provides us with training and testing data-sets. We use a supervised Bayes algorithm to demonstrate an accuracy of better than $66 \%$ of flows and better than $83 \%$ for packets and bytes. Further, we require only the network protocol headers of unknown traffi c for a successful classifi cation stage.

While machine-learning has been used previously for network-traffi c/flow classifi cation e.g., [1], we consider our work to be the fi rst that combines this technique with the use of accurate test and training data-sets.

\section{Experiment}

In order to perform analysis of data using the Naïve Bayes technique, appropriate input data is needed. To do this, we capitalised on trace-data described and categorised in [2]. This classifi ed data was further reduced, and split into 10 equal time intervals each containing around 25,000-65,000 objects (flows). To evaluate the performance of the Naïve Bayes technique, each dataset was used as a training set in turn and evaluated against the remaining datasets, allowing computation of the average accuracy of classifi cation.

Traffi c categories Fundamental to classifi cation work is the idea of classes of traffi c. Throughout this work we use classes of traffi c defi ned as a common group of usercentric applications. Other users of classifi cation may have both simpler defi nitions,

\footnotetext{
${ }^{\star}$ This work was completed when Denis Zuev was employed by Intel Research, Cambridge.

${ }^{\star \star}$ Andrew Moore thanks the Intel Corporation for its generous support of his research fellowship
} 
e.g., Normal versus Malicious, or more complex defi nitions, e.g., the identifi cation of specifi c applications or specifi c TCP implementations.

Described further in [2], we consider the following categories of traffi c: BULK (e.g., ftp), DATABASE (i.e., postgres, etc.), INTERACTIVE (ssh, telnet), MAIL (smtp, etc.), SEVICES (X11, dns), WWW, P2P (e.g., KaZaA, ...), ATTACK (virus and worm attacks), GAMES (Half-Life, ... ), MULTIMEDIA (Windows Media Player, ... ).

Importantly, the characteristics of the traffi c within each category are not necessarily unique. For example, the BULK category which is made up of ftp traffi c, consists of both the control channel, which transfers data in both directions, and the data channel consisting a simplex flow of data for each object transferred. The assignment of categories to applications is an artifi cial grouping that further illustrates that such arbitrary clustering of only-minimally-related traffi c-types is possible with our approach.

Objects and Discriminators Our central object for classifi cation is the flow and for the work presented in this extended-abstract we have limited our defi nition of a flow to being a complete TCP flow - that is all the packets between two hosts for a specific tuple. We restrict ourselves to complete flows, those that start and end validly, e.g., with the fir rst SYN, and the last FIN ACK.

As noted in Section 1, the application of a classifi cation scheme requires the parameterisation of each object to be classifi ed. Using these parameters, the classifi er allocates an object to a class, due to their ability to allow discrimination between classes. We refer to these object-describing parameters as discriminators. In our research we have used 249 different discriminators to describe traffi c flows, these include: flow duration statistics, TCP Port information, payload size statistics, fourier transform of the packet interarrival time discriminators - a complete list is given in [3].

\section{Method}

Machine Learned classifi cation Here we briefly describe the machine learning (ML) approach we take, a trained Naïve Bayes classifi er, along with a number of the refi nements we use. We would direct interested readers to [4] for one of many surveys of all ML techniques.

Several methods exist for classifying data and all of them fall into two broad classes: deterministic and probabilistic classifi cation. As the name suggests, deterministic classifi cation assigns data points to one of a number of mutually-exclusive classes. This is done by considering some metric that defi nes the distance between data points and by defi ning the class boundaries. On the other hand, the probabilistic method classifi es data by assigning it with probabilities of belonging to each class of interest.

We believe that probabilistic classifi cation of Internet traffi c, and our approach in particular, is more suitable given the need to be robust to measurement error, to allow for 
supervised training with pre-classifi ed traffic, to be able to identify similar characteristics of flows after their probabilistic class assignment. We believe that the method be tractable and understood, and be able to cope with the unstable-dynamic nature of Internet traffi $\mathrm{c}$ and that the method allow identification of when the model requires retraining. Additionally, the method needs to be available in a number of implementations.

Naïve Bayesian Classifi er The main approach that is used in this work is the Naive Bayes technique described in [5]. Consider a collection of flows $\mathbf{x}=\left(x_{1}, \ldots, x_{n}\right)$, where each flow $x_{i}$ is described by $m$ discriminators $\left\{d_{1}^{(i)}, \ldots, d_{m}^{(i)}\right\}$ that can take either numeric or discrete values. In the context of the Internet traffi c, $d_{j}^{(i)}$ is a discriminant of flow $x_{i}$, for example it may represent the mean interarrival time of packets in the flow $x_{i}$. In this paper, flows $x_{i}$ belong to exactly one of the mutually-exclusive classes described in Section 2. The supervised Bayesian classifi cation problem deals with building a statistical model that describes each class based on some training data, and where each new flow $y$ receives a probability of getting classifi ed into a particular class according to the Bayes rule below,

$$
p\left(c_{j} \mid y\right)=\frac{p\left(c_{j}\right) f\left(y \mid c_{j}\right)}{\sum_{c_{j}} p\left(c_{j}\right) f\left(y \mid c_{j}\right)}
$$

where $p\left(c_{j}\right)$ denotes the probability of obtaining class $c_{j}$ independently of the observed data, $f\left(y \mid c_{j}\right)$ is the distribution function (or the probability of $y$ given $c_{j}$ ) and the denominator acts as a normalising constant.

The Naiive Bayes technique that is considered in this paper assumes the independence of discriminators $d_{1}, \ldots, d_{m}$ as well as the simple Gaussian behaviour of them. The authors understand that these assumptions are not realistic in the context of the Internet traffi c, but [5] suggest that this model sometimes outperforms certain more complex models.

\section{Naïve Bayes Results}

Our experiments have shown that the Naïve Bayes technique classifi ed on average $66.71 \%$ of the traffi c correctly. Table 1 demonstrates the classifi cation accuracy of this techinique for each class. It can be seen from this table, that SERVICES and BULK are very well classifi ed, with around $90 \%$ of correctly-predicted flows. In comparision to other results, it could be concluded that most discriminator distributions are well separated in the Euclinean space.

At this stage, it is important to note why certain classes performed very poorly. Classes such as GAMES and INTERACTIVE do not contain enough samples, therefore, Naïve Bayes training on these classes is not accurate or realistic. ATTACK flows were often confused with the WWW flows, due to the similarity in discriminators. 


\begin{tabular}{|c|c|c|c|c|c|c|c|c|c|c|}
\hline & WWW & MAIL & BULK & SERV & DB & INT & P2P & ATT & MMEDIA & GAMES \\
Accuracy (\%) & 65.97 & 56.85 & 89.26 & 91.19 & 20.20 & 22.83 & 45.59 & 58.08 & 59.45 & 1.39 \\
\hline Probability (\%) & 98.31 & 90.69 & 90.01 & 35.92 & 61.78 & 7.54 & 4.96 & 1.10 & 32.30 & 100.00 \\
\hline
\end{tabular}

Table 1. Average accuracy of classification of Naïve Bayes technique by class and Probability that the predictive class is the real class.

Alongside accuracy we consider it important to defi ne several other metrics describing the classifi cation technique. Table 1 shows how traffi $\mathrm{c}$ from different classes gets classifi ed - clearly an important measure. However, if a network administrator were to use our tool they would be interested in fi nding out how much trust can be placed in the classifi cation outcome. Table 1 also shows the average probability that the predicted flow class is in fact the real class, e.g., if flow $x_{i}$ has been classifi ed as WWW, a measure of trust gives us a probability that $x_{i}$ is in reality WWW.

A further indication of how well the Naïve Bayes technique performs is to analyse the volume of accurately-classifi ed bytes and packets. The results obtained are: $83.98 \%$ and 83.93\% of packets and bytes, respectively, were correctly classifi ed by the Näve Bayes technique described above. In contrast port-based classifi cation achieved an accuracy of $71.02 \%$ by packet and $69.07 \%$ by bytes (from [2]). Comparing results in this way highlights the signifi cant improvement of our Näve Bayes technique over the portbased classifi cation alone.

\section{Conclusions \& Further Work}

We demonstrate that, in its simplest form, our probabilistic-classifi cation is capable of $67 \%$ accuracy per-flow or better than $83 \%$ accuracy both per-byte and per-packet. We maintain that access to a full-payload trace, the only defi nitive way to characterise network applications, will be limited due to technical and legal restrictions. We illustrate how data gathered without those restrictions may be used as training input for a statistical classifi er which in turn can provide accurate, albeit estimated, classifi cation of header-only trace data.

\section{References}

1. Anthony McGregor et al.: Flow Clustering Using Machine Learning Techniques. In: Proceedings of the Fifth Passive and Active Measurement Workshop (PAM 2004). (2004)

2. Moore, A.W., Papagiannaki, K.: Toward the accurate identification of network applications. In: Passive \& Active Measurement Workshop 2005 (PAM2005), Boston, MA (2005)

3. Moore, A., Zuev, D.: Discriminators for use in fbw-based classification. Technical report, Intel Research, Cambridge (2005)

4. Mitchell, T.: Machine Learning. McGraw Hill (1997)

5. Witten, I.H., Frank, E.: Data Mining. Morgan Kaufmann Publishers (2000) 